\title{
The motivation and status of two-body resonance decays after the LHC Run 2 and beyond
}

\author{
Jeong Han Kim, ${ }^{a}$ Kyoungchul Kong, ${ }^{a}$ Benjamin Nachman ${ }^{b}$ and Daniel Whiteson ${ }^{c}$ \\ ${ }^{a}$ Department of Physics and Astronomy, University of Kansas, \\ Lawrence, KS 66045, U.S.A. \\ ${ }^{b}$ Physics Division, Lawrence Berkeley National Laboratory, \\ Berkeley, CA 94704, U.S.A. \\ ${ }^{c}$ Department of Physics and Astronomy, University of California, \\ Irvine, CA 92697, U.S.A. \\ E-mail: jkim73@nd.edu, kckong@ku.edu, bnachman@cern.ch, daniel@uci.edu
}

\begin{abstract}
Searching for two-body resonance decays is a central component of the high energy physics energy frontier research program. While many of the possibilities are covered when the two bodies are Standard Model (SM) particles, there are still significant gaps. If one or both of the bodies are themselves non-SM particles, there is very little coverage from existing searches. We review the status of two-body searches and motivate the need to search for the missing combinations. It is likely that the search program of the future will be able to cover all possibilities with a combination of dedicated and model agnostic search approaches.
\end{abstract}

KeYWords: Supersymmetry Phenomenology

ARXIV EPRINT: 1907.06659 


\section{Contents}

1 Introduction 1

2 Theory motivation $\quad 2$

$\begin{array}{lll}3 & \text { Current status } & 11\end{array}$

4 Conclusions 12

\section{Introduction}

One of the oldest and most fruitful methods for discovering new particles is to search for resonance structures in invariant mass spectra from the new particle decay products. Most recently, this resulted in the discovery of the Higgs boson [1, 2], but has a long history from the direct observation of the $Z$ boson $[3,4]$, the discovery of the $\Upsilon$ (and thus $b$ quarks) [5], the $J / \psi$ (and thus $c$-quarks) [6, 7], all the way to the $\rho$ meson [8] and likely earlier. This 'bump hunting' continues to be a large component of the search program for the experiments at the Large Hadron Collider (LHC), with about a hundred searches in a multitude of final state configurations [9-13]. Unlike searches targeting more complex final states, for a given topology, two-body resonance searches are only sensitive to two parameters: the mass of the new particle and the production cross-section. ${ }^{1}$ As a result, these searches set powerful constraints on a variety of specific models of physics beyond the Standard Model (BSM).

Given that there have been no confirmed discoveries for new heavy particles since the discovery of the Higgs boson, it is critical to ensure that the complete landscape of two-body resonances is covered by the existing search program. These resonances represent a natural set of targets for searches motivated by experimental sensitivity rather than primarily by theoretical model-building; in this sense they are guides into the unexplored territory of data at the energy frontier, rather than confirmation or rejection of theoretical predictions. The authors of ref. [14] enumerated the possible scenarios and provided physics motivations for $A \rightarrow B C$, where $A$ is a BSM particle and $B$ and $C$ are SM particles. One of our goals in this article is to provide a status update, given that the full Run 2 dataset has been collected and a number of searches have been performed since ref. [14].

While it is critical that dedicated searches targeting specific topologies continue to improve their scope and sensitivity, there is also a growing need for more model agnostic

\footnotetext{
${ }^{1}$ There is also a mild dependence on the width, but this work will mostly consider narrow resonances where the width is small compared to the relevant experimental resolution. Although there are other interesting more-complicated decays, we chose to study two-body resonances, as they provide the simplest and concrete examples. Future work will consider cases where there are undetectable particles (such as neutrinos and dark sectors) as well as multi-body decays.
} 
searches. It may not be possible to have dedicated searches for every possible combination of SM particles for $B$ and $C$, and if either or both of these particles are themselves BSM particles, then the number of possibilities is endless. Recently, there have been a variety of proposals to search for such scenarios in an automated manner using machine learning [15-23]. Our second goal is to extend ref. [14] to cases where $B$ and/or $C$ can be BSM particles to study the motivation and coverage of the complete two-body landscape. This work may help focus on the application of the machine learning-based model agnostic searches.

This paper is organized as follows. Section 2 motivates two-body searches, for both the fully SM and mixed SM/BSM cases. The status of existing experimental searches is presented in section 3. The paper ends with conclusions and outlook in section 4 .

\section{Theory motivation}

Collider searches for resonances are well-motivated by their simplicity and a long history of discoveries. New resonances appear in many extensions of the SM and most of the experimental searches have followed the theoretical models, leading to a variety of searches for a pair of identical objects but rarely for non-identical pairs. However, there is no obvious compelling reason why one should focus only on identical pairs. In fact, the diversity and simple structure of various resonances strongly motivate an experimental program which targets a broad scope and a systematic approach capable of theoretically unanticipated discoveries. Ref. [14] proposed a systematic search program for 2-body resonances, which would consist of searches for resonances in all pairs of SM objects. A majority of 2-body resonances have some indirect theoretical constraints but have received little experimental attention, leaving most of the landscape unexplored and a large potential for unanticipated discovery. It is interesting to note that the lack of these searches is not due to non-existence of theory models as there are models for all possible pairs.

The models described below are illustrative examples of theories which contain such resonances. In some cases, these models may have experimental signatures in other domains or be in conflict with bedrock theoretical arguments. But even in the case where theoretical or indirect experimental constraints exist, there is no replacement for a direct search, which may reveal the existence of an unanticipated particle in tension with current understanding.

In this article, we take a step further and generalize the final state of 2-body resonances to include BSM particles. We present our survey in various tables in this section. We begin with the main classification in table 1, which contains 10 independent decay groups. Each row and column represent how $B$ and $C$ decay after the main decay process, $A \rightarrow B C$. The second cell in the first row $(B)$ and the first column $(C)$ represents a SM particle, while the other three cells represent a BSM particle. These three BSM cells are distinguished based on how they decay: $\mathrm{BSM} \rightarrow \mathrm{SM}_{1} \times \mathrm{SM}_{1}$ (two similar kinds of $\mathrm{SM}$ particles), $\mathrm{BSM} \rightarrow \mathrm{SM}_{1} \times \mathrm{SM}_{2}$ (two different kinds of SM particles), and BSM $\rightarrow$ complex (more complex final states). Our goal is not to provide a complete survey of all available theory models but to catalogue the set of possibilities, providing at least one motivating example for each final state. ${ }^{2}$

\footnotetext{
${ }^{2}$ In nearly every case, there are multiple examples that have been well-studied in dedicated papers (we apologize for not citing your paper!). This is particularly true for signatures that resemble all-hadronic diboson decays [24] or contain di-photon resonances $[25,26]$ due to the excitement over (no longer) excesses reported by ATLAS and CMS.
} 
The left-upper corner of the table (denoted by Group I) reproduces a group of the standard 2-body decays, $A \rightarrow B C$, where $A$ is a BSM particle and $B$ and $C$ are SM particles, as covered in ref. [14]. In this subtable, the column and row are a list of SM particles and each entry corresponds to a mother particle, which would decay into one particle in the column and one particle in the row in the subtable. We show examples of theories that populate the entire landscape of 2-body resonances. $Z^{\prime}$ and $W^{\prime}$ denote additional gauge bosons, $\not R$ represents $R$-parity violating supersymmetry (SUSY), $L^{*}, Q^{*}$ are excited leptons and quarks, respectively, and $T^{\prime}$ and $B^{\prime}$ are a vector-like top and bottom quarks, respectively. $Z_{K K}$ denotes Kaluza-Klein excitation of SM $Z$.

We categorize the rest of table 1 in terms of nine additional subtables, which are denoted by Roman numerals II through X, and present each table in the sequential order. Note that generally we suppress electric charges of each SM particle and focus on the diversity of decay products, although we mention a few interesting examples of such kinds. Similarly we will not distinguish light jets from gluon and generically denote them as $j$ but occasionally we distinguish them for some interesting decays. We denote the bottom quark, and top quark by $b / \bar{b}$ and by $t / \bar{t}$, respectively. The $V$ represents SM gauge bosons $Z$ and $W^{ \pm}$and $H$ is a SM Higgs boson. Throughout the manuscript, a primed particle $X^{\prime}$ represents a BSM particle, whose properties are similar to the corresponding SM particle $X$.

Ref. [14] provide a complete list of possible production mechanisms of two body resonances, including resonant production mode (via the tree-level decay couplings, loopinduced processes involving the decay coupling, or the inclusion of additional couplings to quarks or gluons allowed by the quantum numbers of the resonance), the leading production mode in association with one, two, three, or four Standard Model particles (using the same coupling for production and decay in a four-flavor scheme), the unavoidable existence of a pair production mode. It also notes a possible choice of resonance quantum numbers that does not lead to a pair production mode. However, if one or both of the decay products of $A$ is a BSM particle, $A$ will not be produced as a single resonance at the LHC via the same decay coupling. It requires additional couplings to quarks and gluons, which is not an issue for our discussion in the rest of this study.

In general, various constraints may be imposed on these resonances and could affect the possible production and decay modes. In order to maintain the broadest possible scope, we consider only the most stringent constraints imposed by gauge invariance and Lorentz invariance, as many experimental constraints are dependent on the details of the underlying model and may in principle be evaded. Gauge invariance and Lorentz invariance also dictate the structure of interaction of resonances and SM particles.

Table 2 shows example for $A \rightarrow B C$, where $A$ and $B$ are BSM particles and $C$ is a SM particle, which is the Group II in table 1 . We consider two similar SM particles in the $B$ decays. For example, the $j j$ denotes $B$ decays to two quarks $\left(q \bar{q}, q \bar{q}^{\prime}\right.$ or $\left.q q\right)$, while $\ell \ell$ includes both two opposite-charged leptons $\left(\ell^{+} \ell^{-}\right)$and the same-sign charged leptons $\left(\ell^{+} \ell^{+}\right.$and $\left.\ell^{-} \ell^{-}\right)$and the $V V$ includes the $B$ decays to $g g, \gamma \gamma, \gamma Z, Z Z, W W$, or $Z W$. The $H$ is the observed Higgs boson, $H^{\prime \prime}$ and $H^{\prime}$ are heavy scalars, $A$ is a new pseudo scalar, and $\mathrm{H}^{++}$denotes a doubly-charged scalar particle. The $Q^{\prime}$ represents a generic vector-like quark. $X_{5 / 3}$ and $\pi_{6}^{4 / 3}$ represent a vector-like quark with electric charge $5 / 3$ and 


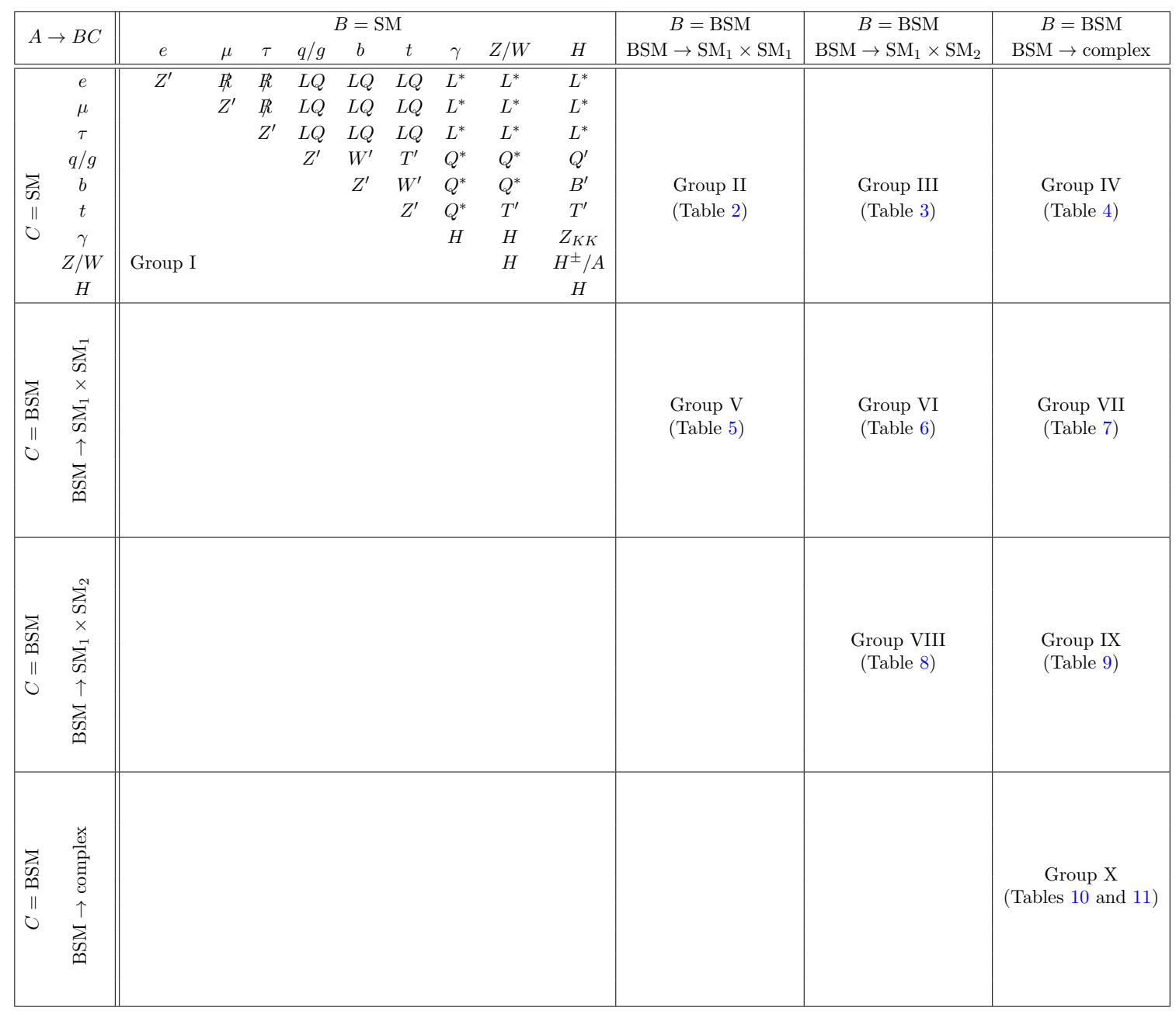

Table 1. Top-level organization of BSM particle $A$ by its two-body decays into $B$ and $C$, showing examples of theoretical motivations for each case. $Z^{\prime}$ and $W^{\prime}$ denote additional gauge bosons, $R$ represents $R$-parity violating SUSY, $L^{*}, Q^{*}$ are excited leptons and quarks, respectively, and $T^{\prime}$ and $B^{\prime}$ are a vector-like top and bottom quarks, respectively. The symbol $Z_{K K}$ denotes Kaluza-Klein excitation of SM $Z$. The SM case in the upper left box is reproduced from ref. [14].

a color-sextet scalar with electric charge $4 / 3$, respectively. Since we consider two similar SM particles, many such examples are either a $Z^{\prime} / W^{\prime}$ or a neutral-heavy scalar.

It is worth noting that when $B$ or $C$ are BSM particles, searches for $A$ are complemented by searches for the $B$ or $C$ particle directly. These approaches are complementary because searches for $A \rightarrow B C$ are sensitive to the coupling between the $A$ and $B / C$ while direct searches for $B / C$ are sensitive to the coupling between $B / C$ and the SM decay products. It is possible that one of these couplings could be sufficiently smaller than the other to render direct searches in one mode insensitive and therefore both search strategies are useful. Figure 1 illustrates the complementarity of direct and indirect searches in the case that $B=C$ and $B \rightarrow q \bar{q}$. The three relevant couplings are between the $A$ particle and quarks $(g(A, q \bar{q}))$, between the $B$ particle $(g(A, B B))$ and between the $B$ particle and 


\begin{tabular}{|c|c|c|c|c|c|}
\hline \multirow{2}{*}{\multicolumn{2}{|c|}{$A \rightarrow B C$}} & \multicolumn{4}{|c|}{$B=\mathrm{BSM}$} \\
\hline & & $\ell \ell$ & $j j$ & $V V$ & $H H$ \\
\hline \multirow{4}{*}{$\mid \begin{array}{c}\sum_{2} \\
\sigma 2 \\
11 \\
0\end{array}$} & $\ell(e, \mu, \tau)$ & $L^{\prime} \rightarrow \ell Z^{\prime}$ & $L^{\prime} \rightarrow \ell Z^{\prime}, N^{\prime} \rightarrow \ell W^{\prime}$ & $L^{\prime} \rightarrow \ell Z^{\prime}, N^{\prime} \rightarrow \ell W^{\prime}$ & $L^{\prime} \rightarrow \ell H^{\prime}$ \\
\hline & $j(b, t, q)$ & $\begin{array}{c}Q^{\prime} \rightarrow j Z^{\prime}, \\
Q^{\prime} \rightarrow j H^{\prime} \\
X_{5 / 3} \rightarrow b H^{++}\end{array}$ & $\begin{aligned} Q^{\prime} & \rightarrow j Z^{\prime}, \\
Q^{\prime} & \rightarrow j H^{\prime} \\
X_{5 / 3} & \rightarrow \bar{b} \pi_{6}^{4 / 3}\end{aligned}$ & $\begin{array}{c}Q^{\prime} \rightarrow j Z^{\prime}, Q^{\prime} \rightarrow j W^{\prime}, \\
Q^{\prime} \rightarrow j H^{\prime}, \\
X_{5 / 3} \rightarrow b H^{++}\end{array}$ & $Q^{\prime} \rightarrow j H^{\prime}$ \\
\hline & $V(W, \gamma, Z)$ & $W^{\prime} \rightarrow W Z^{\prime}$ & $\begin{array}{l}W^{\prime} \rightarrow W Z^{\prime} \\
Z^{\prime} \rightarrow W W^{\prime}\end{array}$ & $\begin{array}{l}W^{\prime} \rightarrow W Z^{\prime} \\
Z^{\prime} \rightarrow W W^{\prime}\end{array}$ & $\begin{array}{c}Z^{\prime} \rightarrow Z H^{\prime}, \\
Z^{\prime} \rightarrow \gamma H^{\prime}\end{array}$ \\
\hline & $H$ & $\begin{array}{c}A \rightarrow H Z^{\prime} \\
H^{\prime \prime} \rightarrow H H^{\prime}\end{array}$ & $\begin{array}{c}A \rightarrow H Z^{\prime}, \\
H^{\prime \prime} \rightarrow H H^{\prime}\end{array}$ & $\begin{array}{c}A \rightarrow H Z^{\prime}, \\
H^{\prime \prime} \rightarrow H H^{\prime}\end{array}$ & $H^{\prime \prime} \rightarrow H H^{\prime}$ \\
\hline
\end{tabular}

Table 2. Example theoretical models for two-body decay of a BSM particle $A$ into a BSM particle $B$ and an SM particle $C$, where the $B$ particle subsequently decays to two similar SM particles (Group II in table 1). The $j j$ denotes $B$ decays to $q \bar{q} / q \bar{q}^{\prime} / q q$, and the $V V$ includes the $B$ decays to $g g, \gamma \gamma, \gamma Z, Z Z, W W$, or $Z W$. The $H$ is the observed Higgs boson, $H^{\prime \prime}$ and $H^{\prime}$ are heavy scalars, $A$ is a new pseudo scalar, and $H^{++}$denotes a doubly-charged scalar particle. The $Q^{\prime}$ represents a generic vector-like quark. In particular, an exotic vector-like quark with electric charge $5 / 3$ is denoted as $X_{5 / 3}$. The $\pi_{6}^{4 / 3}$ is a color-sextet scalar with electric charge $4 / 3$.

quarks $(g(B, q \bar{q}))$. When $m_{B} \ll m_{A}$, so that the $B$ decay products are contained inside a single jet, the inclusive dijet search sets strong limits on $A$ production. These limits would be significantly weaker when $m_{B}$ is not sufficiently small for its decay products to be contained inside an $R=0.4$ jet, which is the jet radius used by both the ATLAS and CMS inclusive dijet searches. For $m_{A}=2 \mathrm{TeV}$, the current limit on $g(A, q \bar{q})$ is about $0.1[27,28]$. For moderate (not contained) $m_{B}$, this means that there is strong sensitivity up to $g(A, B B) \sim g(A, q \bar{q})$. For larger $g(A, B B)$, there would be stronger sensitivity from a direct search that targets the full $A \rightarrow B B$ topology, e.g. a search for two jets with substructure and not just a search for two generic quark/gluon jets. The coupling $g(A, q \bar{q}) \sim 0.1$ at $m_{A} \sim 2 \mathrm{TeV}$ corresponds to a cross section limit of about $0.1 \mathrm{pb}$. The direct search for $B$ sets limits of about $1 \mathrm{nb}$ at $m_{B}=300 \mathrm{GeV}$, which corresponds to $g(B, q \bar{q}) \approx 0.2[29,30]$. Therefore, the direct $B$ search is not sensitive to the $B$ 's produced from $A$ production. However, the direct search for $B$ can be competitive when $g(A, q \bar{q})$ is small. In particular, if $g(A, q \bar{q})<0.1$, then the direct search for $A$ is insensitive, but if $g(B, q \bar{q})>0.2$, then the $B$ search is sensitive. In general, this is also true for other final states and we expect significant improvement possible with a dedicated search for $A \rightarrow B C \rightarrow(S M S M)(S M S M)$, especially in the parameter space where $g(A, B C) \gtrsim g(A, S M S M)$ and $g(B, S M S M) /$ $g(C, S M S M)$ is not too large.

In next group (Group III in table 1 ), we consider the case where $B$ decays to two different types of SM particles. Unlike table 2 where many examples are either a $Z^{\prime}$ or a neutral-heavy scalar, in this category of table 3, many examples of $B$ are either a vectorlike quark, a charged scalar or a $W^{\prime}$, since we consider two different particles. Once the spin of $B$ is fixed, we can easily find the spin nature of $A$, for a given SM particle for $C$. 


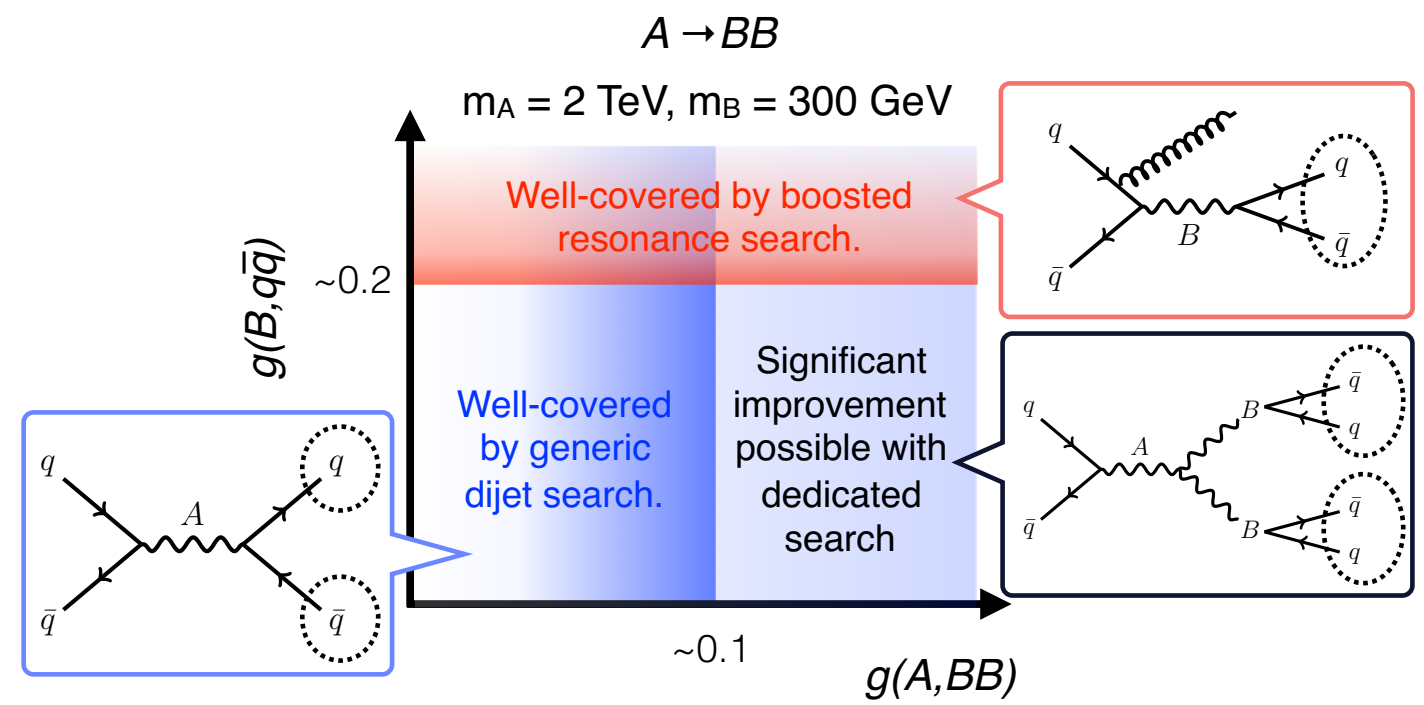

Figure 1. An illustration of the complementarity of the search for $A$ (inclusive dijet resonance search) and the search for $B$ (boosted resonance search). Dotted circles indicate hadronic activity that will likely be mostly captured by one (potentially large-radius) jet. When $m_{A}=2 \mathrm{TeV}$ and $m_{B}=300 \mathrm{GeV}$, the inclusive dijet search likely has reduced sensitivity to $A \rightarrow B B$ because the $B$ decay products are not well-contained inside a single small-radius jet. Therefore, when $g(A, B B) \gtrsim$ $g(A, q \bar{q}) \sim 0.1$, gains are possible for a dedicated search.

\begin{tabular}{|c|c|c|c|c|c|}
\hline & \multirow[b]{2}{*}{$A \rightarrow B C$} & \multicolumn{4}{|c|}{$B=\mathrm{BSM}$} \\
\hline & & $\begin{array}{c}t Z, t H, W b, \\
t g, \text { or } t \gamma\end{array}$ & $\begin{array}{c}b Z, b H, W t \\
b g, \text { or } b \gamma\end{array}$ & $\ell Z, \ell \gamma$, or $\ell H$ & $\begin{array}{c}\gamma W, Z W, H W \\
\text { or } t b\end{array}$ \\
\hline \multirow{4}{*}{$\begin{array}{l}\sum_{i=1} \\
\| \\
0\end{array}$} & $\ell$ & $L Q \rightarrow \ell T^{\prime}$ & $L Q \rightarrow \ell B^{\prime}$ & $H^{\prime} \rightarrow \ell L^{\prime}$ & $N \rightarrow \ell W^{\prime}, N \rightarrow \ell H^{+}$ \\
\hline & $j(b, t, q)$ & $\begin{aligned} W^{\prime} & \rightarrow b T^{\prime} \\
Z^{\prime} & \rightarrow t T^{\prime}\end{aligned}$ & $\begin{aligned} W^{\prime} & \rightarrow t B^{\prime} \\
Z^{\prime} & \rightarrow b B^{\prime}\end{aligned}$ & $L Q \rightarrow j L^{\prime}$ & $\begin{array}{c}B^{\prime} \rightarrow t W^{\prime}, T^{\prime} \rightarrow b H^{+}, \\
X_{5 / 3} \rightarrow t W^{\prime}, B^{\prime} \rightarrow t H^{-} \\
X_{5 / 3} \rightarrow t H^{+}\end{array}$ \\
\hline & $V(W, \gamma, Z)$ & $\begin{aligned} B^{\prime} & \rightarrow W T^{\prime} \\
X_{-7 / 3} & \rightarrow W X_{-4 / 3}\end{aligned}$ & $\begin{array}{c}T^{\prime} \rightarrow W B^{\prime}, \\
X_{8 / 3} \rightarrow W X_{5 / 3}\end{array}$ & $L^{\prime \prime} \rightarrow Z L^{\prime}$ & $\begin{array}{l}H^{+} \rightarrow \gamma W^{\prime} \\
Z^{\prime} \rightarrow W H^{+}\end{array}$ \\
\hline & $H$ & $T^{\prime \prime} \rightarrow H T^{\prime}$ & $B^{\prime \prime} \rightarrow H B^{\prime}$ & $L^{\prime \prime} \rightarrow H L^{\prime}$ & $\begin{aligned} W^{\prime \prime} & \rightarrow H W^{\prime} \\
H^{+\prime} & \rightarrow H H^{+}\end{aligned}$ \\
\hline
\end{tabular}

Table 3. Example (Group III in table 1) for $A \rightarrow B C$, where $A$ and $B$ are BSM particles and $C$ is a SM particle. The $B$ decays to two different SM particles are considered. The $L Q$ is a leptoquark carrying both baryon and lepton numbers, and the $N$ is a right-handed heavy neutrino. Exotic vector-like quarks with electric charges $-7 / 3,-4 / 3,5 / 3$, and $8 / 3$ are denoted as $X_{-7 / 3}, X_{-4 / 3}$, $X_{5 / 3}$, and $X_{8 / 3}$ respectively. The $H^{+}$and $H^{-}$are new charged scalar particles.

Here the $L Q$ is a leptoquark carrying both baryon and lepton numbers, and the $N$ is a right-handed heavy neutrino. Exotic vector-like quarks with electric charges $-7 / 3,-4 / 3$, $5 / 3$, and $8 / 3$ are denoted as $X_{-7 / 3}, X_{-4 / 3}, X_{5 / 3}$, and $X_{8 / 3}$ respectively. The $H^{+}$and $H^{-}$ are new charged scalar particles. 


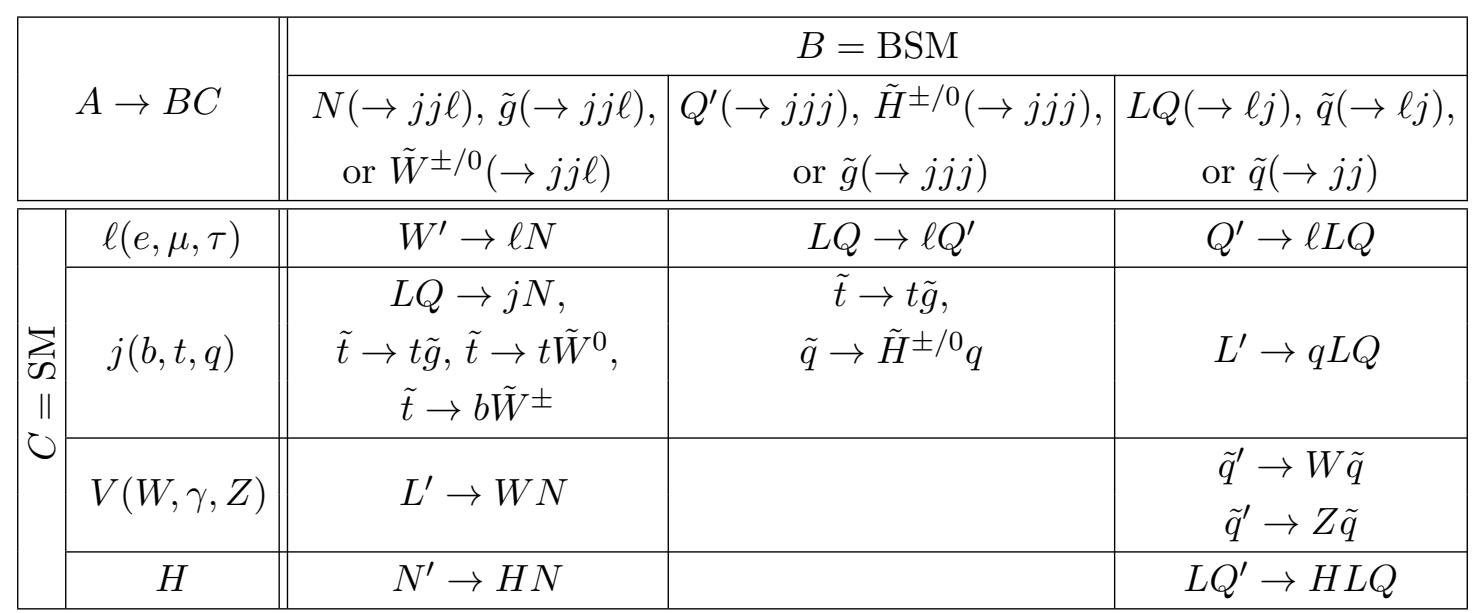

Table 4. Example (Group IV in table 1) for $A \rightarrow B C$, where $A$ and $B$ are BSM particles and $C$ is a SM particle. The $B$ decays to more complex final states are considered. The $L^{\prime}$ is a vector-like lepton, and $N$ denotes a right-handed heavy neutrino which can decay to $N \rightarrow W^{\prime(*)} \ell \rightarrow j j \ell$. The $Q^{\prime}$ represents a generic vector-like quark decaying to $Q^{\prime} \rightarrow W^{\prime(*)} j \rightarrow j j j$. The $\tilde{t}$ denotes a stop, and the $\tilde{g}$ is a gluino which decays through RPV couplings [31, 32]. The $\tilde{W}^{0}$ and $\tilde{W}^{ \pm}$are neutral and charged winos respectively. The neutral and charged Higgsinos, $\tilde{H}^{0}$ and $\tilde{H}^{ \pm}$, can decay to $\tilde{H}^{ \pm / 0} \rightarrow j \tilde{q}^{*} \rightarrow j j j$ through RPV couplings.

The next example (Group IV) shown in table 4 is similar to the previous case (Group III), but we consider the case where $B$ decays in a more complicated way. Many examples that we present are due to 3-body decay or decays through $R$-parity violating interaction (RPV) in supersymmetry. For instance, a right-handed heavy neutrino $N$ could decay to $N \rightarrow W^{\prime(*)} \ell \rightarrow j j \ell$ via an off-shell $W^{\prime}$. Similarly, a generic vector-like quark $Q^{\prime}$ could decay to $Q^{\prime} \rightarrow W^{\prime(*)} j \rightarrow j j j$. In table 4 , the $\tilde{t}$ denotes a stop, and the $\tilde{g}$ is a gluino which decays through RPV couplings $[31,32]$. The $\tilde{W}^{0}$ and $\tilde{W}^{ \pm}$are neutral and charged winos respectively. The neutral and charged Higgsinos, $\tilde{H}^{0}$ and $\tilde{H}^{ \pm}$, can decay to $\tilde{H}^{ \pm / 0} \rightarrow j \tilde{q}^{*} \rightarrow$ jjj through RPV couplings.

The next group (Group $\mathrm{V}$ in table 1) presented in table 5 is the first example of $A \rightarrow B C$ decay, where $A, B$ and $C$ are all BSM particles. We consider that each of $B$ and $C$ decays to similar kinds of SM particles. As discussed in table 2 , the $j j$ denotes two quark-system of all possible flavor combinations. Here the $V V$ includes $\gamma \gamma, \gamma Z, Z Z$, $Z W, \gamma W$, or $W W$. We abbreviate the decays $G_{\Theta \phi_{I}}^{\prime} \equiv G^{\prime} \rightarrow \Theta \phi_{I}, G_{\Theta \Theta}^{\prime} \equiv G^{\prime} \rightarrow \Theta \Theta$, $\Theta \equiv \Theta \rightarrow G^{\prime} G^{\prime}$, and $\phi_{I} \equiv \phi_{I} \rightarrow \Theta G^{\prime}$ where $G^{\prime}, \Theta$, and $\phi_{I}$ denote a coloron, color-octet scalar, and a singlet scalar, respectively [33, 34]. The extended Two-Higgs Doublet Model with a real singlet (2HDMS) $[35,36]$ allows for the decay of a CP-even heavy scalar into light scalars, abbreviated as $2 H \equiv H^{\prime \prime} \rightarrow H^{\prime} H^{\prime}$. We also consider heavy $Z$ boson decays $Z^{\prime} \equiv Z^{\prime} \rightarrow W^{\prime+} W^{\prime-}, H^{+} H^{-}, W^{\prime \pm} H^{\mp}$, or $H^{++} H^{--}$where $H^{ \pm}$and $H^{ \pm \pm}$are singly- and doubly-charged scalars.

The next two Groups, VI and VII, in tables 6 and 7 are similar to Groups III and IV, respectively. In both cases, $C$ decays to similar kind SM particles, while $B$ decays to two different kinds (Group VI, table 5) or more complex final state (Group VII, table 6). 


\begin{tabular}{|c|c|c|c|c|c|c|}
\hline \multirow{2}{*}{\multicolumn{2}{|c|}{$A \rightarrow B C$}} & \multicolumn{5}{|c|}{$B=\mathrm{BSM}$} \\
\hline & & \multirow{2}{*}{$\frac{j j}{\Theta \Theta, Z^{\prime}}$} & \multirow{2}{*}{$\begin{array}{l}g g \\
\phi_{I}\end{array}$} & \multirow{2}{*}{$\frac{V V}{2 \mathrm{H}, Z^{\prime}}$} & \multirow{2}{*}{$\frac{\ell \ell}{2 \mathrm{H}}$} & \multirow{2}{*}{$\begin{array}{c}H H \\
2 \mathrm{H}\end{array}$} \\
\hline \multirow{5}{*}{$\begin{array}{l}\sum_{\|} \\
n \\
0 \\
0\end{array}$} & $j j$ & & & & & \\
\hline & $g g$ & & $G_{\Theta \Theta}^{\prime}$ & $2 \mathrm{H}, G_{\Theta \phi_{I}}^{\prime}$ & $2 \mathrm{H}$ & $2 \mathrm{H}$ \\
\hline & $V V$ & & & $2 \mathrm{H}, Z^{\prime}$ & $2 \mathrm{H}$ & $2 \mathrm{H}$ \\
\hline & $\ell \ell$ & & & & $2 \mathrm{H}$ & $2 \mathrm{H}$ \\
\hline & $H H$ & & & & & $2 \mathrm{H}$ \\
\hline
\end{tabular}

Table 5. Example (Group V in table 1) for $A \rightarrow B C$, where $A, B$ and $C$ are BSM particles. Each of $B$ and $C$ decays to similar kinds of SM particles. The $j j$ denotes a diquark with all possible flavor combinations. Here the $V V$ includes $\gamma \gamma, \gamma Z, Z Z, Z W, \gamma W$, or $W W$. We abbreviate the decays $G_{\Theta \phi_{I}}^{\prime} \equiv G^{\prime} \rightarrow \Theta \phi_{I}, G_{\Theta \Theta}^{\prime} \equiv G^{\prime} \rightarrow \Theta \Theta, \Theta \equiv \Theta \rightarrow G^{\prime} G^{\prime}$, and $\phi_{I} \equiv \phi_{I} \rightarrow \Theta G^{\prime}$ where $G^{\prime}$, $\Theta$, and $\phi_{I}$ denote a coloron, color-octet scalar, and a singlet scalar respectively [33, 34]. Extended Two-Higgs Doublet Model with a real singlet (2HDMS) [35, 36] allow for the decay of a CP-even heavy scalar into light scalars, abbreviated as $2 H \equiv H^{\prime \prime} \rightarrow H^{\prime} H^{\prime}$. We also consider heavy $Z$ boson decays $Z^{\prime} \equiv Z^{\prime} \rightarrow W^{\prime+} W^{\prime-}, H^{+} H^{-}, W^{\prime \pm} H^{\mp}$, or $H^{++} H^{--}$where $H^{ \pm}$and $H^{ \pm \pm}$are singly- and doubly-charged scalars.

\begin{tabular}{|c|c||c|c|c|c|}
\hline \multicolumn{3}{|c||}{$A \rightarrow B C$} & \multicolumn{4}{c|}{$B=\mathrm{BSM}$} \\
\cline { 2 - 6 } & $\begin{array}{c}t Z, t H, W b, \\
t g, \text { or } t \gamma\end{array}$ & $\begin{array}{c}b Z, b H, W t, \\
b g, \text { or } b \gamma\end{array}$ & $\ell Z, \ell \gamma$, or $\ell H$ & $H W$ \\
\hline \hline \multirow{2}{*}{\begin{tabular}{c}
\multirow{2}{*}{$j$} \\
$\|$
\end{tabular}} & $T^{\prime \prime} \rightarrow Z^{\prime} T^{\prime}$ & $B^{\prime \prime} \rightarrow Z^{\prime} B^{\prime}$ & $L^{\prime \prime} \rightarrow Z^{\prime} L^{\prime}$ & $\begin{array}{c}Z^{\prime} \rightarrow H^{ \pm} W^{\prime \mp} \\
Z^{\prime} \rightarrow W^{\prime+} W^{\prime-}\end{array}$ \\
\cline { 2 - 6 } & $V V$ & $T^{\prime \prime} \rightarrow S^{\prime} T^{\prime}$ & $B^{\prime \prime} \rightarrow S^{\prime} B^{\prime}$ & $L^{\prime \prime} \rightarrow S^{\prime} L^{\prime}$ & \\
\cline { 2 - 6 } & $H H$ & $T^{\prime \prime} \rightarrow S^{\prime} T^{\prime}$ & $B^{\prime \prime} \rightarrow S^{\prime} B^{\prime}$ & $L^{\prime \prime} \rightarrow S^{\prime} L^{\prime}$ & \\
\hline
\end{tabular}

Table 6. Example (Group VI in table 1) for $A \rightarrow B C$, where $A, B$ and $C$ are BSM particles. The $C$ decays to two similar SM particles, while the $B$ decays to two different kinds of SM particles. The $j j$ denotes a diquark with all possible flavor combinations. The $V V$ includes $g g, \gamma \gamma, \gamma Z, Z Z$, or $W W$.

\begin{tabular}{|c|c|c|c|c|c|}
\hline \multirow{2}{*}{\multicolumn{2}{|c|}{$A \rightarrow B C$}} & \multicolumn{4}{|c|}{$B=\mathrm{BSM}$} \\
\hline & & jjl & 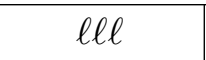 & $t W W$ & $j j j$ \\
\hline $\begin{array}{l}\sum_{n} \\
0 \\
0 \\
0\end{array}$ & $j j, V V$, or $H H$ & $\begin{aligned} N^{\prime} & \rightarrow Z^{\prime} N, \\
N^{\prime} & \rightarrow H^{\prime} N, \\
L^{\prime} & \rightarrow W^{\prime} N\end{aligned}$ & $\begin{array}{l}L^{\prime \prime} \rightarrow Z^{\prime} L^{\prime} \\
L^{\prime \prime} \rightarrow H^{\prime} L^{\prime}\end{array}$ & $\begin{aligned} X_{8 / 3}^{\prime} & \rightarrow Z^{\prime} X_{8 / 3} \\
X_{8 / 3}^{\prime} & \rightarrow H^{\prime} X_{8 / 3}\end{aligned}$ & $\tilde{q} \rightarrow \tilde{H}^{+} \tilde{q}^{\prime}$ \\
\hline
\end{tabular}

Table 7. Example (Group VII in table 1) for $A \rightarrow B C$, where $A, B$ and $C$ are BSM particles. The $C$ decays to two similar SM particles, while the $B$ decays to more complex final states. The $j j$ denotes a diquark with all possible flavor combinations, and $V V$ includes $\gamma \gamma, \gamma Z, Z Z$, or $W W$. The $N$ denotes a right-handed heavy neutrino which decays to $N \rightarrow W^{\prime(*)} \ell \rightarrow j j \ell$. The $L^{\prime}$ represents a generic vector-like lepton decaying to $L^{\prime} \rightarrow Z^{\prime(*)} \ell \rightarrow \ell \ell \ell$. 


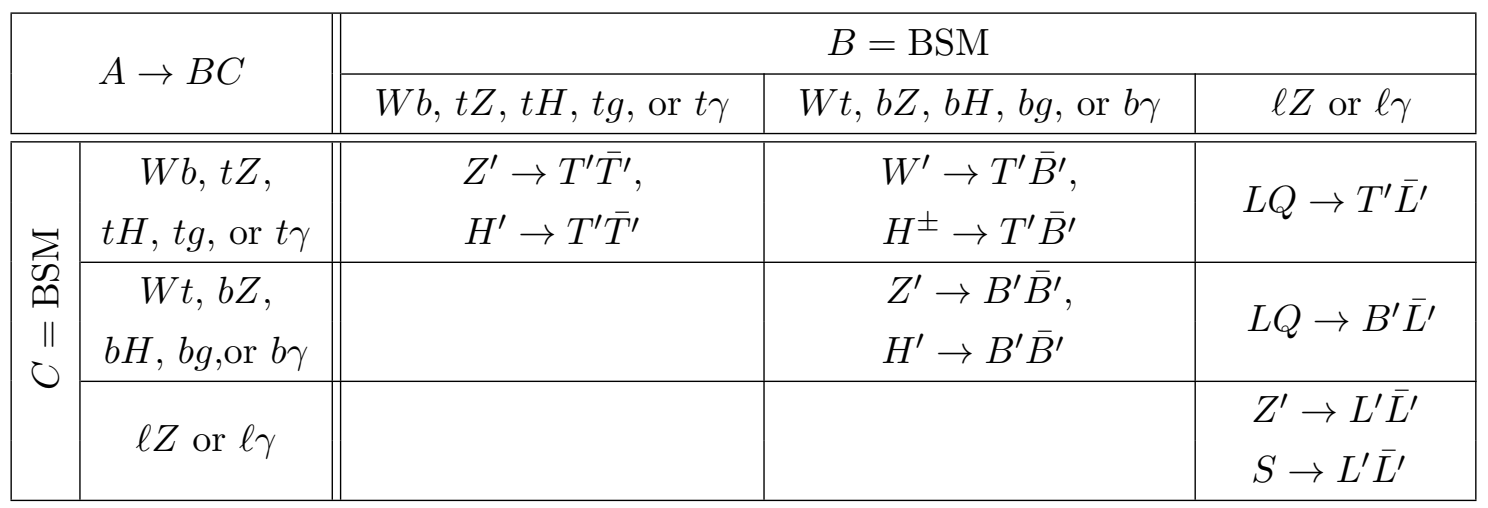

Table 8. Example (Group VIII in table 1) for $A \rightarrow B C$, where $A, B$, and $C$ are BSM particles. Each of $B$ and $C$ decays to different kinds of SM particles. This table shows the resonant productions (via either a new gauge boson or a new scalar) of new fermions.

\begin{tabular}{|c|c|c|c|}
\hline & \multirow{2}{*}{$A \rightarrow B C$} & \multicolumn{2}{|c|}{$B=\mathrm{BSM}$} \\
\hline & & jjl & $\ell \ell \ell$ \\
\hline \multirow{3}{*}{ 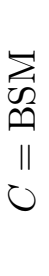 } & $\begin{array}{c}W b, t Z \\
t H, t g, \text { or } t \gamma\end{array}$ & $L Q \rightarrow T^{\prime} N$ & $L Q \rightarrow T^{\prime} L^{\prime}$ \\
\hline & $\begin{array}{c}W t, b Z, \\
b H, b g, \text { or } b \gamma\end{array}$ & $L Q \rightarrow B^{\prime} N$ & $L Q \rightarrow B^{\prime} L^{\prime}$ \\
\hline & $\ell Z$ or $\ell \gamma$ & $W^{\prime} \rightarrow L^{\prime} N$ & $Z^{\prime} \rightarrow L^{\prime} L^{\prime}$ \\
\hline
\end{tabular}

Table 9. Example (Group IX in table 1) for $A \rightarrow B C$, where $A, B$ and $C$ are BSM particles. The $C$ decays to two different SM particles, while the $B$ decays to more complex final states. The $N$ denotes a right-handed heavy neutrino which decays to $N \rightarrow W^{\prime(*)} \ell \rightarrow j j \ell$. The $L^{\prime}$ represents a generic vector-like lepton decaying to $L^{\prime} \rightarrow Z^{\prime(*)} \ell \rightarrow \ell \ell \ell$. The $T^{\prime}, B^{\prime}$, and $L^{\prime}$ decays are the same as presented in table 5 .

In table 8 , we present examples for $A \rightarrow B C$, where $A, B$, and $C$ are BSM particles and both $B$ and $C$ decay to different kinds of SM particles (Group VIII in table 1). As mentioned for table 3, many examples of two different SM decay products are decays of vector-like fermions. Therefore an obvious example for Group VIII would be the resonant production of two vector-like fermions (via either a new gauge boson or a new scalar).

The last two Groups (IX and X) involve more complex decays. In table 9 , only $B$ follows the complex decays, while in table 10 both $B$ and $C$ give the complex final states. We consider 3-body decays of a right-handed heavy neutrino or a vector-like quark for such examples.

As an alternative example for $\mathrm{X}$, we provide various coloron decays in table 11 . In this example, the $j$ includes a $t, b$, and light-flavor quarks. The $G^{\prime}, \Theta$, and $\phi_{I}$ denote a coloron, color-octet scalar, and a singlet scalar respectively. The three particles naturally arise in a 'renormalizable coloron model' [33, 34]. It is interesting that a simple coloron model provides such diverse signatures, depending on the mass spectrum. 


\begin{tabular}{|c|c|c|c|c|}
\hline \multirow{2}{*}{\multicolumn{2}{|c|}{$A \rightarrow B C$}} & \multicolumn{3}{|c|}{$B=\mathrm{BSM}$} \\
\hline & & $N(\rightarrow j j \ell)$ & $Q^{\prime}(\rightarrow j j j)$ & $L Q(\rightarrow \ell j)$ \\
\hline \multirow{3}{*}{$\begin{array}{l}\sum_{n} \\
n \\
\| \\
0 \\
0\end{array}$} & $N(\rightarrow j j \ell)$ & $Z^{\prime} \rightarrow N \bar{N}$ & $Q^{\prime \prime} \rightarrow N Q^{\prime}$ & \\
\hline & $Q^{\prime}(\rightarrow j j j)$ & & $Z^{\prime} \rightarrow Q^{\prime} \bar{Q}^{\prime}$ & $L^{\prime} \rightarrow Q^{\prime} L Q$ \\
\hline & $L Q(\rightarrow \ell j)$ & & & $Z^{\prime} \rightarrow L Q \overline{L Q}$ \\
\hline
\end{tabular}

Table 10. Example (Group $\mathrm{X}$ in table 1) for $A \rightarrow B C$, where $A, B$ and $C$ are BSM particles. Both $B$ and $C$ decay to more complex final states. The $N$ denotes a right-handed heavy neutrino which can decay into $N \rightarrow W^{\prime(*)} \ell \rightarrow j j \ell$. The $Q^{\prime}$ represents a generic vector-like quark decaying to $Q^{\prime} \rightarrow W^{\prime(*)} j \rightarrow j j j$.

\begin{tabular}{|c|c|c|c|c|c|c|c|c|}
\hline \multirow{2}{*}{\multicolumn{2}{|c|}{$A \rightarrow B C$}} & \multicolumn{7}{|c|}{$B=\mathrm{BSM}$} \\
\hline & & $g g j j$ & ggjjjj & $W W j j$ & $\gamma Z j j$ & $Z Z j j$ & $H j j$ & $H j j j j$ \\
\hline \multirow{7}{*}{$\begin{array}{l}\sum_{n} \\
\text { pी } \\
\| \\
\|\end{array}$} & $g g j j$ & & $G^{\prime} \rightarrow \Theta \phi_{I}$ & $G^{\prime} \rightarrow \Theta \phi_{I}$ & $G^{\prime} \rightarrow \Theta \phi_{I}$ & $G^{\prime} \rightarrow \Theta \phi_{I}$ & & $G^{\prime} \rightarrow \Theta \phi_{I}$ \\
\hline & ggjjjj & & $G^{\prime} \rightarrow \Theta \Theta$ & $G^{\prime} \rightarrow \Theta \Theta$ & $G^{\prime} \rightarrow \Theta \Theta$ & $G^{\prime} \rightarrow \Theta \Theta$ & $G^{\prime} \rightarrow \Theta \phi_{I}$ & $G^{\prime} \rightarrow \Theta \Theta$ \\
\hline & $W W j j$ & & & $G^{\prime} \rightarrow \Theta \Theta$ & $G^{\prime} \rightarrow \Theta \Theta$ & $G^{\prime} \rightarrow \Theta \Theta$ & $G^{\prime} \rightarrow \Theta \phi_{I}$ & $G^{\prime} \rightarrow \Theta \Theta$ \\
\hline & $\gamma Z j j$ & & & & $G^{\prime} \rightarrow \Theta \Theta$ & $G^{\prime} \rightarrow \Theta \Theta$ & $G^{\prime} \rightarrow \Theta \phi_{I}$ & $G^{\prime} \rightarrow \Theta \Theta$ \\
\hline & $Z Z j j$ & & & & & $G^{\prime} \rightarrow \Theta \Theta$ & $G^{\prime} \rightarrow \Theta \phi_{I}$ & $G^{\prime} \rightarrow \Theta \Theta$ \\
\hline & $H j j$ & & & & & & & $G^{\prime} \rightarrow \Theta \phi_{I}$ \\
\hline & $H j j j j$ & & & & & & & $G^{\prime} \rightarrow \Theta \Theta$ \\
\hline
\end{tabular}

Table 11. Example (Group $\mathrm{X}$ in table 1) for $A \rightarrow B C$, where $A, B$ and $C$ are BSM particles, where both $B$ and $C$ decay to more complex final states. The $j$ includes a $t, b$, and light-flavor quarks. The $G^{\prime}, \Theta$, and $\phi_{I}$ denote a coloron, color-octet scalar, and a singlet scalar respectively [33, 34]. So we could call all these entries as 'a renormalizable coloron model'. It is interesting that a simple coloron model provides such diverse signatures, depending on the mass spectrum.

Finally we make a brief remark on combining different groups. In Groups II and III, $A$ and $B$ are BSM resonances and $C$ is SM particle. Since $C$ is a SM particle, we can classify $A$ and $B$ based on the spin of $C$. Some examples are shown in table 12 for Groups II and III. Any pair, $F F, F V$ etc only indicates Lorentz structure and they could have different (QED, QCD) charges. $C$ could be $F, V$ or $H$, and $B$ (2-body resonance) will decay into any possible pair of $C$ s. The spin of $A$ will be determined, once the spin of $B$ is chosen. All primed particles are BSM particles. In principle, this classification could include Group IV but would be more complicated. $X$ represents either $S$ or $V$.

Similarly we can combine Groups V, VI and VIII, and show generic presentation of Lorentz structure in table 13. Here $A, B$ and $C$ are BSM resonances and both $B$ and $C$ could decay into any possible pair of $F, V$ or $H$. The spin of $A$ will determined, once the spins of $B$ and $C$ are chosen. In principle, this classification could include Groups VII, IX and X but would be more complicated. The point of this exercise in tables 12 and 13 is that we can find an example of any resonance, once we specify (QED, QCD) quantum numbers and Lorentz structure. 


\begin{tabular}{|c||c|c|c|c|c|c|}
\hline$A \rightarrow B C$ & $B \rightarrow F F$ & $B \rightarrow V V$ & $B \rightarrow H H$ & $B \rightarrow F V$ & $B \rightarrow F H$ & $B \rightarrow V H$ \\
\hline \hline $\mathrm{C}=\mathrm{F}$ & $F^{\prime} \rightarrow F X^{\prime}$ & $F^{\prime} \rightarrow F X^{\prime}$ & $F^{\prime} \rightarrow F X^{\prime}$ & $X^{\prime} \rightarrow F F^{\prime}$ & $X^{\prime} \rightarrow F F^{\prime}$ & $F^{\prime} \rightarrow F X^{\prime}$ \\
\hline $\mathrm{C}=\mathrm{V}$ & $V^{\prime \prime} \rightarrow V X^{\prime}$ & $S^{\prime \prime} \rightarrow V X^{\prime}$ & $S^{\prime \prime} \rightarrow V X^{\prime}$ & $F^{\prime \prime} \rightarrow V F^{\prime}$ & $F^{\prime \prime} \rightarrow V F^{\prime}$ & $X^{\prime \prime} \rightarrow V X^{\prime}$ \\
\hline $\mathrm{C}=\mathrm{H}$ & $X^{\prime \prime} \rightarrow H X^{\prime}$ & $X^{\prime \prime} \rightarrow H X^{\prime}$ & $X^{\prime \prime} \rightarrow H X^{\prime}$ & $F^{\prime \prime} \rightarrow H F^{\prime}$ & $F^{\prime \prime} \rightarrow H F^{\prime}$ & $X^{\prime \prime} \rightarrow H X^{\prime}$ \\
\hline
\end{tabular}

Table 12. Example (Groups II and III in table 1) for $A \rightarrow B C$ purely based on Lorentz structure, where $A$ and $B$ are BSM resonances and $C$ is SM particle. The corresponding (QED, QCD) charges need to be understood properly, depending on quantum charges of the involved particles. Any pair, $F F, F V$ etc only indicates Lorentz structure and they could have different (QED, QCD) charges. $C$ could be $F, V$ or $H$, and $B$ (2 body resonance) will decay into any possible pair of $F, V$ or $H$. The spin of $A$ will be determined, once the spin of $B$ is chosen. All primed particles are BSM particles. In principle, this classification could include III but would be more complicated. $X$ is either a scalar $(S)$ or a vector $(V)$.

\begin{tabular}{|l||c|c|c|c|c|c|}
\hline$A \rightarrow B C$ & $B \rightarrow F F$ & $B \rightarrow V V$ & $B \rightarrow H H$ & $B \rightarrow F V$ & $B \rightarrow F H$ & $B \rightarrow V H$ \\
\hline \hline$C \rightarrow F F$ & $X^{\prime \prime \prime} \rightarrow X^{\prime \prime} X^{\prime}$ & $X^{\prime \prime \prime} \rightarrow X^{\prime \prime} X^{\prime}$ & $X^{\prime \prime \prime} \rightarrow X^{\prime \prime} X^{\prime}$ & $F^{\prime \prime} \rightarrow X^{\prime} F^{\prime}$ & $F^{\prime \prime} \rightarrow X^{\prime} F^{\prime}$ & $X^{\prime \prime \prime} \rightarrow X^{\prime \prime} X^{\prime}$ \\
\hline$C \rightarrow V V$ & & $X^{\prime \prime \prime} \rightarrow X^{\prime \prime} X^{\prime}$ & $X^{\prime \prime \prime} \rightarrow X^{\prime \prime} X^{\prime}$ & $F^{\prime \prime} \rightarrow X^{\prime} F^{\prime}$ & $F^{\prime \prime} \rightarrow X^{\prime} F^{\prime}$ & $X^{\prime \prime \prime} \rightarrow X^{\prime \prime} X^{\prime}$ \\
\hline$C \rightarrow H H$ & & & $X^{\prime \prime \prime} \rightarrow X^{\prime \prime} X^{\prime}$ & $F^{\prime \prime} \rightarrow X^{\prime} F^{\prime}$ & $F^{\prime \prime} \rightarrow X^{\prime} F^{\prime}$ & $X^{\prime \prime \prime} \rightarrow X^{\prime \prime} X^{\prime}$ \\
\hline$C \rightarrow F V$ & & & & $X^{\prime} \rightarrow F^{\prime} F^{\prime \prime}$ & $X^{\prime} \rightarrow F^{\prime} F^{\prime \prime}$ & $F^{\prime \prime} \rightarrow F^{\prime} X^{\prime}$ \\
\hline$C \rightarrow F H$ & & & & & $X^{\prime \prime \prime} \rightarrow X^{\prime \prime} X^{\prime}$ & $F^{\prime \prime} \rightarrow F^{\prime} X^{\prime}$ \\
\hline$C \rightarrow V H$ & & & & & & $X^{\prime \prime \prime} \rightarrow X^{\prime \prime} X^{\prime}$ \\
\hline
\end{tabular}

Table 13. Example (Groups V, VI and VIII in table 1) for $A \rightarrow B C$ purely based on Lorentz structure, where $A, B$ and $C$ are BSM resonances. The corresponding (QED, QCD) charges need to be understood properly, depending on quantum charges of the involved particles. Any pair, $F F$, $F V$ etc only indicates Lorentz structure and they could have different QED/QCD charges. Both $B$ and $C$ could decay into any possible pair of $F, V$ or $H$. The spin of $A$ will determined, once the spins of $B$ and $C$ are chosen. In principle, this classification could include VII, IX and X.

\section{Current status}

ATLAS and CMS have an impressive and extensive search program that already includes many of the possibilities described in the previous sections. In particular, a few more of the $A \rightarrow \mathrm{SM} \times \mathrm{SM}$ possibilities described in ref. [14] are now covered by Run 2 searches. Table 14 describes the current coverage to both the $\mathrm{SM} \times \mathrm{SM}$ and more generic 2-body resonances cases using published searches based on Run 2 data.

The first important feature of table 14 is that many of the $\mathrm{SM} \times \mathrm{SM}$ possibilities are still uncovered, most notably the final states involving a lepton and a quark/gluon or Higgs boson. The second important feature of table 14 is that when one or both of $B / C$ are BSM, most of the possibilities are uncovered. In some cases, such as $B / C \rightarrow$ quarks/gluons, there is some complementarity with direct $B / C$ searches (see section 2 ). This is also true when $B$ or $C$ decay into leptons or vector bosons, but the $B / C$ search limits are much weaker due to the low production cross-section of vector boson fusion at the LHC and the available center-of-mass energy at current and previous lepton colliders.

Despite a large number of existing searches, table 14 combined with section 2 indicate that there are many well-motivated possibilities that are currently uncovered. New searches can close these gaps in coverage and ensure broad sensitivity to BSM possibilities. 


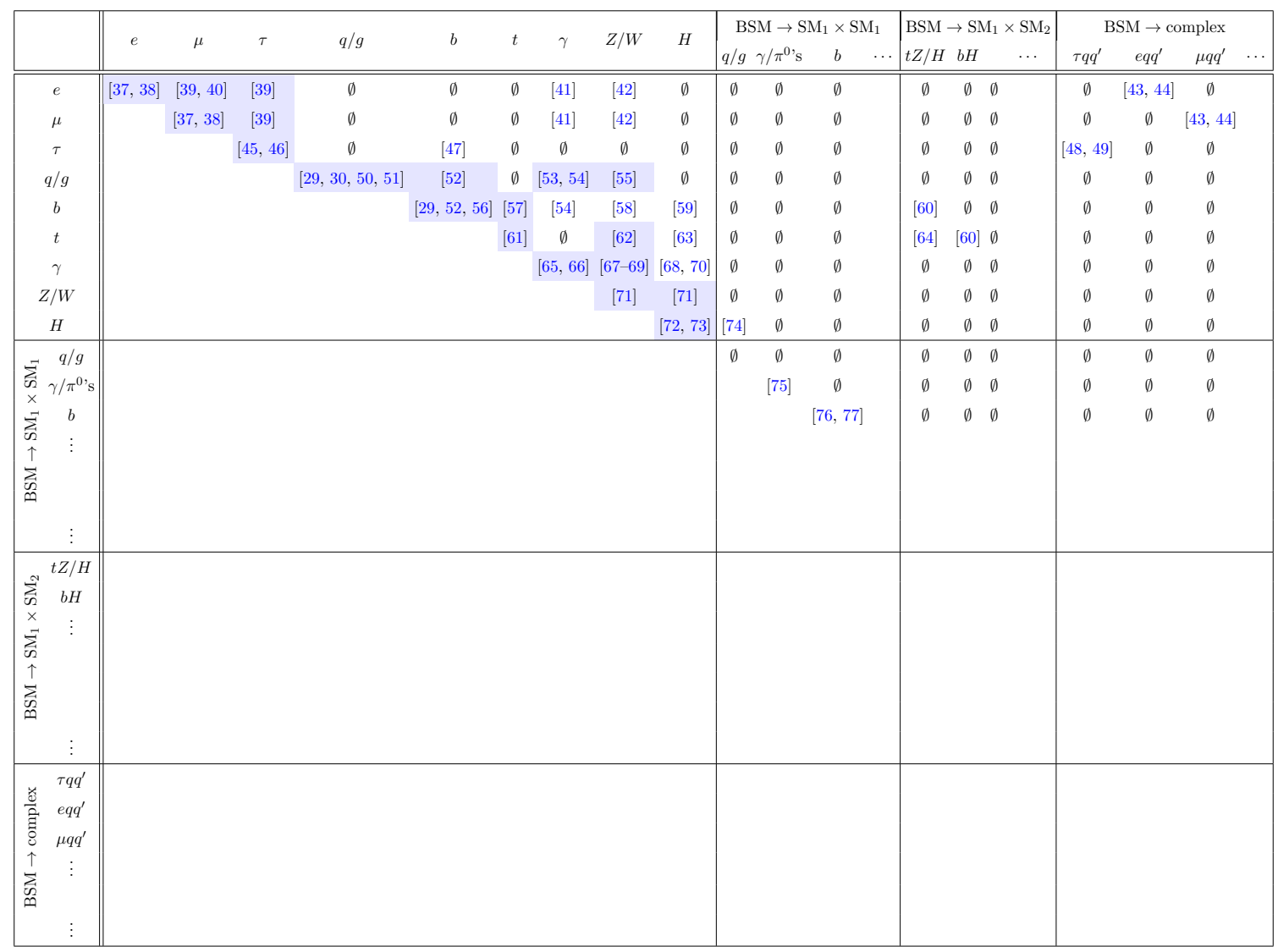

Table 14. References to existing searches for two-body resonances, where one decay product is from the first column and one is from the first row. Only the most recent searches are considered. The box $\mathrm{BSM} \rightarrow \mathrm{SM}_{1} \times \mathrm{SM}_{2}$ represents cases where the primary resonance decays to a BSM particle, which itself decays into two SM particles that are not the same. Colored cells indicate searches that were covered by $\sqrt{s}=8 \mathrm{TeV}$ searches reported in ref. [14].

\section{Conclusions}

Two-body resonance searches are a cornerstone of the LHC search program. While the current experimental coverage is broad, there are many well-motivated scenarios that are all or partially uncovered. We have catalogued the set of possibilities, providing at least one motivating example for each final state. Given the lack of significant excess at the LHC and the lack of a unique theory to guide the search program, now is the time to consider diversifying the experimental sensitivity. Organizing the possibilities by final state provides a way forward.

While the traditional search program will be able to accommodate many of the possibilities described earlier, there are not enough resources to consider all potential final states. Therefore, dedicated searches will likely need to be complimented with more model agnostic searches. Machine learning methods may be able to automate this approach and solve significant statistical challenges like large trails factors $[15,16]$. In particular, techniques such as neural networks can readily analyze high-dimensional spaces and approaches with 
cross-validation can avoid over-training. A variety of advanced machine learning methods have been proposed recently [15-20, 20-23, 78-80] which can be trained without any particular signal model. As a result, these algorithms would be broadly sensitive to new resonances and offer a powerful complement to dedicated searches for particular topologies. Many of these techniques can be trained directly on data [15-20, 20,21, 78-80] and so can naturally be incorporated into the usual bump hunting framework. While these tools will not be more sensitive than a dedicated search on any particular topology, they can be simultaneously sensitive to a wide variety of resonances. For example, in the case that $m_{A} \neq m_{B} \neq m_{C}$, a traditional search would pay a significant 'look elsewhere effect' by scanning over $m_{A}, m_{B}$, and $m_{C}$. In contrast, methods like those proposed in refs. [15, 16] only pay the trails factor for a scan in $m_{A}$ and let a neural network discover structure that could point to a localized feature from resonances $B$ and $C$. As another example, a method trained with photons and jets may be sensitive to diphoton, dijet, and mixed photon-jet resonances all at once while traditional methods typically focus on one topology at a time. These methods are in their infancy, but have a great potential to fill in gaps in the existing search program.

This work has focused on two-body decays into visible final states. Future work will consider more complex cases where there are undetectable particles, such as neutrinos and dark sectors, as well as multi-body decays.

The LHC experiments have and will continue to collect rich datasets that may contain answers to key questions about the fundamental properties of nature. Many well-motivated fundamental theories have provided guiding principles to analyses these data. However, a more diversified perspective will be required to full exploit the data - in fact, there may be something new already hiding in the existing datasets!

\section{Acknowledgments}

This work was supported by the U.S. Department of Energy, Office of Science under contract DE-AC02-05CH11231, DE-SC0017988 and DE-SC0019474.

Open Access. This article is distributed under the terms of the Creative Commons Attribution License (CC-BY 4.0), which permits any use, distribution and reproduction in any medium, provided the original author(s) and source are credited.

\section{References}

[1] ATLAS collaboration, Observation of a new particle in the search for the Standard Model Higgs boson with the ATLAS detector at the LHC, Phys. Lett. B 716 (2012) 1 [arXiv: 1207.7214] [INSPIRE].

[2] CMS collaboration, Observation of a New Boson at a Mass of $125 \mathrm{GeV}$ with the CMS Experiment at the LHC, Phys. Lett. B 716 (2012) 30 [arXiv:1207.7235] [INSPIRE].

[3] UA1 collaboration, Experimental Observation of Lepton Pairs of Invariant Mass Around 95-GeV/c² at the CERN SPS Collider, Phys. Lett. B 126 (1983) 398 [InSPIRE]. 
[4] UA2 collaboration, Evidence for $Z^{0} \rightarrow e^{+} e^{-}$at the CERN $\bar{p} p$ Collider, Phys. Lett. B 129 (1983) 130 [INSPIRE].

[5] S.W. Herb et al., Observation of a Dimuon Resonance at 9.5-GeV in 400-GeV Proton-Nucleus Collisions, Phys. Rev. Lett. 39 (1977) 252 [INSPIRE].

[6] E598 collaboration, Experimental Observation of a Heavy Particle J, Phys. Rev. Lett. 33 (1974) 1404 [INSPIRE].

[7] SLAC-SP-017 collaboration, Discovery of a Narrow Resonance in $e^{+} e^{-}$Annihilation, Phys. Rev. Lett. 33 (1974) 1406 [InSPIRE].

[8] J. Button, G.R. Kalbfleisch, G.R. Lynch, B.C. Maglić, A.H. Rosenfeld and M.L. Stevenson, Pion-Pion Interaction in the Reaction barp $+p \rightarrow 2 \pi^{+}+2 \pi^{-}+n \pi^{0}$, Phys. Rev. 126 (1962) 1858 [INSPIRE].

[9] ATLAS collaboration, Exotic physics searches, https://twiki.cern.ch/twiki/bin/view/AtlasPublic/ExoticsPublicResults, (2019).

[10] ATLAS collaboration, Higgs and diboson searches, https://twiki.cern.ch/twiki/bin/view/AtlasPublic/HDBSPublicResults, (2019).

[11] CMS collaboration, CMS exotica public physics results, https://twiki.cern.ch/twiki/bin/view/CMSPublic/PhysicsResultsEXO, (2019).

[12] CMS collaboration, CMS beyond-two-generations (b2g) public physics results, https://twiki.cern.ch/twiki/bin/view/CMSPublic/PhysicsResultsB2G, (2019).

[13] LHCb collaboration, Publications of the qcd, electroweak and exotica working group, http://lhcbproject.web.cern.ch/lhcbproject/Publications/LHCbProjectPublic/ Summary_QEE.html, (2019).

[14] N. Craig, P. Draper, K. Kong, Y. Ng and D. Whiteson, The unexplored landscape of two-body resonances, Acta Phys. Polon. B 50 (2019) 837 [arXiv:1610.09392] [INSPIRE].

[15] J.H. Collins, K. Howe and B. Nachman, Extending the search for new resonances with machine learning, Phys. Rev. D 99 (2019) 014038 [arXiv: 1902.02634] [INSPIRE].

[16] J.H. Collins, K. Howe and B. Nachman, Anomaly Detection for Resonant New Physics with Machine Learning, Phys. Rev. Lett. 121 (2018) 241803 [arXiv:1805. 02664] [INSPIRE].

[17] M. Farina, Y. Nakai and D. Shih, Searching for New Physics with Deep Autoencoders, arXiv: 1808.08992 [INSPIRE].

[18] T. Heimel, G. Kasieczka, T. Plehn and J.M. Thompson, QCD or What?, SciPost Phys. 6 (2019) 030 [arXiv: 1808.08979] [INSPIRE].

[19] O. Cerri, T.Q. Nguyen, M. Pierini, M. Spiropulu and J.-R. Vlimant, Variational Autoencoders for New Physics Mining at the Large Hadron Collider, JHEP 05 (2019) 036 [arXiv: 1811.10276] [INSPIRE].

[20] T.S. Roy and A.H. Vijay, A robust anomaly finder based on autoencoder, arXiv:1903.02032 [INSPIRE].

[21] J. Hajer, Y.-Y. Li, T. Liu and H. Wang, Novelty Detection Meets Collider Physics, arXiv:1807.10261 [INSPIRE].

[22] A. De Simone and T. Jacques, Guiding New Physics Searches with Unsupervised Learning, Eur. Phys. J. C 79 (2019) 289 [arXiv:1807.06038] [INSPIRE]. 
[23] R.T. D'Agnolo and A. Wulzer, Learning New Physics from a Machine, Phys. Rev. D 99 (2019) 015014 [arXiv: 1806.02350] [INSPIRE].

[24] ATLAS collaboration, Search for high-mass diboson resonances with boson-tagged jets in proton-proton collisions at $\sqrt{s}=8 \mathrm{TeV}$ with the ATLAS detector, JHEP 12 (2015) 055 [arXiv: 1506.00962] [INSPIRE].

[25] CMS collaboration, Search for Resonant Production of High-Mass Photon Pairs in Proton-Proton Collisions at $\sqrt{s}=8$ and 13 TeV, Phys. Rev. Lett. 117 (2016) 051802 [arXiv: 1606. 04093] [INSPIRE].

[26] ATLAS collaboration, Search for resonances in diphoton events at $\sqrt{s}=13 \mathrm{TeV}$ with the ATLAS detector, JHEP 09 (2016) 001 [arXiv:1606.03833] [INSPIRE].

[27] ATLAS collaboration, Search for New Phenomena in Dijet Events using $139 \mathrm{fb}^{-1}$ of pp collisions at $\sqrt{s}=13$ TeV collected with the ATLAS Detector, ATLAS-CONF-2019-007 (2019).

[28] CMS collaboration, Searches for dijet resonances in pp collisions at $\sqrt{s}=13 \mathrm{TeV}$ using the 2016 and 2017 datasets, CMS-PAS-EXO-17-026 (2018).

[29] ATLAS collaboration, Search for low-mass resonances decaying into two jets and produced in association with a photon using pp collisions at $\sqrt{s}=13 \mathrm{TeV}$ with the ATLAS detector, Phys. Lett. B 795 (2019) 56 [arXiv:1901.10917] [INSPIRE].

[30] CMS collaboration, Search for low mass vector resonances decaying into quark-antiquark pairs in proton-proton collisions at $\sqrt{s}=13 \mathrm{TeV}$, JHEP 01 (2018) 097 [arXiv:1710.00159] [INSPIRE].

[31] R. Barbier et al., R-parity violating supersymmetry, Phys. Rept. 420 (2005) 1 [hep-ph/0406039] [INSPIRE].

[32] J.A. Evans and Y. Kats, LHC Coverage of RPV MSSM with Light Stops, JHEP 04 (2013) 028 [arXiv: 1209.0764] [INSPIRE].

[33] Y. Bai and B.A. Dobrescu, Collider Tests of the Renormalizable Coloron Model, JHEP 04 (2018) 114 [arXiv: 1802.03005] [INSPIRE].

[34] Y. Bai, S. Lu and Q.-F. Xiang, Hexapod Coloron at the LHC, JHEP 08 (2018) 200 [arXiv: 1805.09815] [INSPIRE].

[35] G.C. Branco, P.M. Ferreira, L. Lavoura, M.N. Rebelo, M. Sher and J.P. Silva, Theory and phenomenology of two-Higgs-doublet models, Phys. Rept. 516 (2012) 1 [arXiv:1106.0034] [INSPIRE].

[36] C.-Y. Chen, M. Freid and M. Sher, Next-to-minimal two Higgs doublet model, Phys. Rev. D 89 (2014) 075009 [arXiv: 1312.3949] [INSPIRE].

[37] ATLAS collaboration, Search for high-mass dilepton resonances using $139 \mathrm{fb}^{-1}$ of pp collision data collected at $\sqrt{s}=13 \mathrm{TeV}$ with the ATLAS detector, ATLAS-CONF-2019-001 (2019).

[38] CMS collaboration, Search for high-mass resonances in dilepton final states in proton-proton collisions at $\sqrt{s}=13 \mathrm{TeV}$, JHEP 06 (2018) 120 [arXiv: 1803.06292] [INSPIRE].

[39] ATLAS collaboration, Search for lepton-flavor violation in different-flavor, high-mass final states in pp collisions at $\sqrt{s}=13 \mathrm{TeV}$ with the ATLAS detector, Phys. Rev. D 98 (2018) 092008 [arXiv: 1807.06573] [INSPIRE]. 
[40] CMS collaboration, Search for lepton-flavor violating decays of heavy resonances and quantum black holes to e $\mu$ final states in proton-proton collisions at $\sqrt{s}=13$ TeV, JHEP 04 (2018) 073 [arXiv: 1802.01122] [INSPIRE].

[41] CMS collaboration, Search for excited leptons in $\ell \ell \gamma$ final states in proton-proton collisions at $\sqrt{s}=13 \mathrm{TeV}$, JHEP 04 (2019) 015 [arXiv: 1811.03052] [INSPIRE].

[42] CMS collaboration, Search for heavy neutral leptons in events with three charged leptons in proton-proton collisions at $\sqrt{s}=13$ TeV, Phys. Rev. Lett. 120 (2018) 221801 [arXiv: 1802.02965] [INSPIRE].

[43] CMS collaboration, Search for a heavy right-handed $W$ boson and a heavy neutrino in events with two same-flavor leptons and two jets at $\sqrt{s}=13$ TeV, JHEP 05 (2018) 148 [arXiv: 1803.11116] [INSPIRE].

[44] CMS collaboration, Search for a heavy composite Majorana neutrino in the final state with two leptons and two quarks at $\sqrt{s}=13$ TeV, Phys. Lett. B 775 (2017) 315 [arXiv: 1706.08578] [INSPIRE].

[45] ATLAS collaboration, Search for additional heavy neutral Higgs and gauge bosons in the ditau final state produced in $36 \mathrm{fb}^{-1}$ of pp collisions at $\sqrt{\mathrm{s}}=13$ TeV with the ATLAS detector, JHEP 01 (2018) 055 [arXiv:1709.07242] [INSPIRE].

[46] CMS collaboration, Search for heavy resonances decaying to tau lepton pairs in proton-proton collisions at $\sqrt{s}=13 \mathrm{TeV}$, JHEP 02 (2017) 048 [arXiv: 1611.06594] [INSPIRE].

[47] CMS collaboration, Search for a singly produced third-generation scalar leptoquark decaying to a $\tau$ lepton and a bottom quark in proton-proton collisions at $\sqrt{s}=13$ TeV, JHEP 07 (2018) 115 [arXiv: 1806.03472] [INSPIRE].

[48] CMS collaboration, Search for heavy neutrinos and third-generation leptoquarks in hadronic states of two $\tau$ leptons and two jets in proton-proton collisions at $\sqrt{s}=13$ TeV, JHEP 03 (2019) 170 [arXiv:1811.00806] [INSPIRE].

[49] CMS collaboration, Search for third-generation scalar leptoquarks and heavy right-handed neutrinos in final states with two tau leptons and two jets in proton-proton collisions at $\sqrt{s}=13 \mathrm{TeV}$, JHEP 07 (2017) 121 [arXiv:1703.03995] [INSPIRE].

[50] ATLAS collaboration, Search for new phenomena in dijet mass and angular distributions from pp collisions at $\sqrt{s}=13$ TeV with the ATLAS detector, Phys. Lett. B 754 (2016) 302 [arXiv: 1512.01530] [INSPIRE].

[51] CMS collaboration, Search for narrow and broad dijet resonances in proton-proton collisions at $\sqrt{s}=13$ TeV and constraints on dark matter mediators and other new particles, JHEP 08 (2018) 130 [arXiv:1806.00843] [INSPIRE].

[52] ATLAS collaboration, Search for resonances in the mass distribution of jet pairs with one or two jets identified as b-jets in proton-proton collisions at $\sqrt{s}=13 \mathrm{TeV}$ with the ATLAS detector, Phys. Rev. D 98 (2018) 032016 [arXiv: 1805.09299] [InSPIRE].

[53] ATLAS collaboration, Search for new phenomena in high-mass final states with a photon and a jet from $p p$ collisions at $\sqrt{s}=13$ TeV with the ATLAS detector, Eur. Phys. J. C 78 (2018) 102 [arXiv: 1709.10440] [INSPIRE].

[54] CMS collaboration, Search for excited quarks of light and heavy flavor in $\gamma+$ jet final states in proton-proton collisions at $\sqrt{s}=13$ TeV, Phys. Lett. B 781 (2018) 390 [arXiv: 1711.04652] [INSPIRE]. 
[55] CMS collaboration, Search for massive resonances decaying into $W W, W Z, Z Z, q W$ and $q Z$ with dijet final states at $\sqrt{s}=13 \mathrm{TeV}$, Phys. Rev. D 97 (2018) 072006 [arXiv: 1708.05379] [INSPIRE].

[56] CMS collaboration, Search for low-mass resonances decaying into bottom quark-antiquark pairs in proton-proton collisions at $\sqrt{s}=13 \mathrm{TeV}$, Phys. Rev. D 99 (2019) 012005 [arXiv: 1810.11822] [INSPIRE].

[57] ATLAS collaboration, Search for vector-boson resonances decaying to a top quark and bottom quark in the lepton plus jets final state in pp collisions at $\sqrt{s}=13 \mathrm{TeV}$ with the ATLAS detector, Phys. Lett. B 788 (2019) 347 [arXiv:1807.10473] [INSPIRE].

[58] ATLAS collaboration, Search for single production of vector-like quarks decaying into Wb in pp collisions at $\sqrt{s}=13 \mathrm{TeV}$ with the ATLAS detector, JHEP 05 (2019) 164 [arXiv: 1812.07343] [INSPIRE].

[59] ATLAS collaboration, Search for single production of a vector-like B quark decaying into a bottom quark and a Higgs boson which decays into a pair of photons, ATLAS-CONF-2018-024 (2018).

[60] CMS collaboration, Search for a $W^{\prime}$ boson decaying to a vector-like quark and a top or bottom quark in the all-jets final state, JHEP 03 (2019) 127 [arXiv:1811.07010] [INSPIRE].

[61] ATLAS collaboration, Search for heavy particles decaying into a top-quark pair in the fully hadronic final state in pp collisions at $\sqrt{s}=13 \mathrm{TeV}$ with the ATLAS detector, Phys. Rev. D 99 (2019) 092004 [arXiv: 1902.10077] [INSPIRE].

[62] CMS collaboration, Search for single production of vector-like quarks decaying to a top quark and a $W$ boson in proton-proton collisions at $\sqrt{s}=13$ TeV, Eur. Phys. J. C 79 (2019) 90 [arXiv: 1809.08597] [INSPIRE].

[63] CMS collaboration, Search for electroweak production of a vector-like quark decaying to a top quark and a Higgs boson using boosted topologies in fully hadronic final states, JHEP 04 (2017) 136 [arXiv: 1612.05336] [INSPIRE].

[64] CMS collaboration, Search for a heavy resonance decaying to a top quark and a vector-like top quark in the lepton + jets final state in pp collisions at $\sqrt{s}=13$ TeV, Eur. Phys. J. C 79 (2019) 208 [arXiv: 1812.06489] [INSPIRE].

[65] ATLAS collaboration, Search for new phenomena in high-mass diphoton final states using $37 \mathrm{fb}^{-1}$ of proton-proton collisions collected at $\sqrt{s}=13 \mathrm{TeV}$ with the ATLAS detector, Phys. Lett. B 775 (2017) 105 [arXiv:1707.04147] [INSPIRE].

[66] CMS collaboration, Search for physics beyond the standard model in high-mass diphoton events from proton-proton collisions at $\sqrt{s}=13$ TeV, Phys. Rev. D 98 (2018) 092001 [arXiv: 1809.00327] [INSPIRE].

[67] ATLAS collaboration, Search for heavy resonances decaying to a $Z$ boson and a photon in pp collisions at $\sqrt{s}=13 \mathrm{TeV}$ with the ATLAS detector, Phys. Lett. B 764 (2017) 11 [arXiv: 1607.06363] [INSPIRE].

[68] ATLAS collaboration, Search for heavy resonances decaying to a photon and a hadronically decaying $Z / W / H$ boson in pp collisions at $\sqrt{s}=13 \mathrm{TeV}$ with the ATLAS detector, Phys. Rev. D 98 (2018) 032015 [arXiv: 1805.01908] [inSPIRE]. 
[69] CMS collaboration, Search for $Z \gamma$ resonances using leptonic and hadronic final states in proton-proton collisions at $\sqrt{s}=13 \mathrm{TeV}$, JHEP 09 (2018) 148 [arXiv:1712.03143] [INSPIRE].

[70] CMS collaboration, Search for narrow $H \gamma$ resonances in proton-proton collisions at $\sqrt{s}=13$ TeV, Phys. Rev. Lett. 122 (2019) 081804 [arXiv: 1808.01257] [INSPIRE].

[71] ATLAS collaboration, Combination of searches for heavy resonances decaying into bosonic and leptonic final states using $36 \mathrm{fb}^{-1}$ of proton-proton collision data at $\sqrt{\mathrm{s}}=13 \mathrm{TeV}$ with the ATLAS detector, Phys. Rev. D 98 (2018) 052008 [arXiv: 1808. 02380] [inSPIRE].

[72] ATLAS collaboration, Search for resonant and non-resonant Higgs boson pair production in the $b \bar{b} \tau^{+} \tau^{-}$decay channel in pp collisions at $\sqrt{s}=13 \mathrm{TeV}$ with the ATLAS detector, Phys. Rev. Lett. 121 (2018) 191801 [Erratum ibid. 122 (2019) 089901] [arXiv:1808.00336] [INSPIRE].

[73] ATLAS collaboration, Search for pair production of Higgs bosons in the $b \bar{b} b \bar{b}$ final state using proton-proton collisions at $\sqrt{s}=13 \mathrm{TeV}$ with the ATLAS detector, JHEP 01 (2019) 030 [arXiv: 1804.06174] [INSPIRE].

[74] ATLAS collaboration, A search for resonances decaying into a Higgs boson and a new particle $X$ in the $X H \rightarrow q q b b$ final state with the ATLAS detector, Phys. Lett. B 779 (2018) 24 [arXiv:1709.06783] [INSPIRE].

[75] ATLAS collaboration, A search for pairs of highly collimated photon-jets in pp collisions at $\sqrt{s}=13 \mathrm{TeV}$ with the ATLAS detector, Phys. Rev. D 99 (2019) 012008 [arXiv:1808.10515] [INSPIRE].

[76] ATLAS collaboration, Search for long-lived particles produced in pp collisions at $\sqrt{s}=13$ TeV that decay into displaced hadronic jets in the ATLAS muon spectrometer, Phys. Rev. D 99 (2019) 052005 [arXiv: 1811.07370] [InSPIRE].

[77] ATLAS collaboration, Search for long-lived neutral particles in pp collisions at $\sqrt{s}=13 \mathrm{TeV}$ that decay into displaced hadronic jets in the ATLAS calorimeter, Eur. Phys. J. C 79 (2019) 481 [arXiv: 1902.03094] [INSPIRE].

[78] J.A. Aguilar-Saavedra, J.H. Collins and R.K. Mishra, A generic anti-QCD jet tagger, JHEP 11 (2017) 163 [arXiv:1709.01087] [INSPIRE].

[79] A. Blance, M. Spannowsky and P. Waite, Adversarially-trained autoencoders for robust unsupervised new physics searches, JHEP 10 (2019) 047 [arXiv: 1905.10384] [INSPIRE].

[80] B.M. Dillon, D.A. Faroughy and J.F. Kamenik, Uncovering latent jet substructure, Phys. Rev. D 100 (2019) 056002 [arXiv: 1904.04200] [INSPIRE]. 